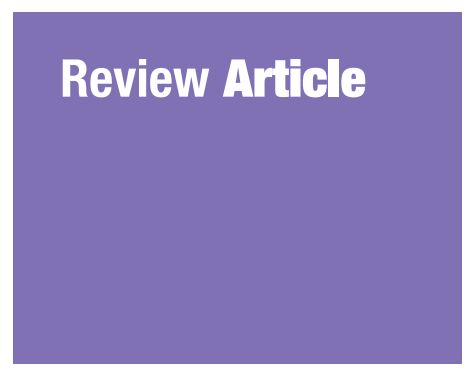

Submitted: 24 Feb 2019

Accepted: 23 Dec 2019

Online: 30 Apr 2020

\section{An Assessment of the Movement and Function of Children with Specific Learning Disabilities: A Review of Five Standardised Assessment Tools}

\author{
Nur Sakinah Baharudin, Dzalani Harun, Masne Kadar
}

Occupational Therapy Programme, Center for Rehabilitation and Special Needs Studies, Faculty of Health Sciences, Universiti Kebangsaan Malaysia, Kuala Lumpur, Malaysia

To cite this article: Baharudin NS, Harun D, Kadar M. An assessment of the movement and function of children with specific learning disabilities: a review of five standardised assessment tools. Malays $J$ Med Sci. 2020;27(2):21-36. https://doi.org/10.21315/mjms2020.27.2.3

To link to this article: https://doi.org/10.21315/mjms2020.27.2.3

\begin{abstract}
Various standardised assessment tools have been used to evaluate children with disabilities. However, assessment tools that provide information on the movement and function of children with specific learning disabilities (SLD) are still limited. This article provides a narrative review of the characteristics of five movement and/or function assessment tools. The strengths and limitations of the tools will be highlighted. Empirical studies on the assessment tools used are reviewed based on three criteria: (i) standardised tools; (ii) assessment of movement and/or function; (iii) applicability to children with SLD ranging from 4-17 years of age and widely used in practice. The following instruments have been included as they have been found to fulfil the criteria: (i) the Bruininks-Oseretsky test of motor proficiency-2 (BOT-2); (ii) the movement assessment battery for children-2 (MABC-2); (iii) the pediatric balance scale (PBS); (iv) the Vineland adaptive behaviour scale-II (VABS-II) and (v) the pediatric evaluation of disability inventory-computerised adaptive test (PEDI-CAT). The article presents the characteristics, strengths and limitations of five standardised assessment tools that are currently in use, which measure the movement and/or function of children with SLD. This article concludes with a discussion of recommendations for the best approaches to evaluating the movement and functional abilities of children with SLD.
\end{abstract}

Keywords: assessment tool, children, function, movement, specific learning disabilities

\section{Introduction}

A specific learning disability (SLD) is a type of neurodevelopmental disorder. SLD is defined by the fifth edition of the Diagnostic and Statistical Manual of Mental Disorders (DSM-5) (1) as consisting of four key elements: i) characterised by having constant difficulty in learning and using one or more academic domains (i.e. reading comprehension, spelling, writing difficulties, mathematical reasoning) for at least six months although target skill interventions have been given; ii) the concerned academic skills are below what is expected at the individual's age, which impairs functioning in school, at work and in activities of daily living (ADL); iii) SLD will be diagnosed at the age of onset during school-age or some people may appear the disorder when higher-level skills are demanded and iv) excluding those who have intellectual disabilities, visual or hearing impairments, mental disorders, neurological disorders, psychosocial-difficulties, language differences and who lack proficiency in the language of academic instruction (1).

Standardised assessment tools provide limited information on the movement and/or functional skill performance of children with 
SLD. Previous studies have mainly focused on assessing literacy problems in children such as reading (2) and other academic achievements (3-4). In addition, such studies have been developed and used by Western populations. The results of such studies have shown that movement and functional skill performance in school-aged children is lacking. Notably, the tasks item referring to function skills are less relevant to the cultural context in Malaysia. Therefore, it is necessary to select an appropriate movement and functions assessment for children with SLD in Malaysia.

The review focuses on evaluative measures to assess movement and functional performance in children with SLD. Aside from academic difficulties, children with SLD have often been found to exhibit clumsy and awkward movements while performing physical exercise (5). They show deficits in movement performance, such as in gross motor (6), fine motor (7), balance $(8,9)$ and functional skills (10). Insights into movement and functional performance of children with SLD are essential for health professionals and teachers to manage movement difficulty in these children. Therefore, the professionals involved are responsible for measuring motor proficiency in children with SLD (10-11).

Movement refers to the observable act of moving and demonstrates a change in the position of any part of the body (12). The performance of movement can be measured using motor skills to produce an output. Locomotors skills (e.g. running and hopping), manipulative or object control skills (e.g. catching and throwing) and balance skills (e.g. balancing and twisting) are fundamental movement skills required of school-aged children with SLD to deal with everyday life activities (13). Examples of movement tools used to assess children are the Bruininks-Oseretsky of motor proficiency test, second edition (BOT-2) (14) and the movement assessment battery for children, second edition (MABC-2) (15).

Function refers to performing an action or activities considered necessary for everyday life (16). For example, routine activities of daily living (ADL) include eating, grooming, toileting, dressing and functional mobility (16). There are several functional assessment tools available that measure ADL skills for children, such as the Vineland adaptive behaviour scale, second edition (VABS-II) (17) and the pediatric evaluation of disability inventory-computerised adaptive test (PEDI-CAT) (18).
This review explores some standardised tools for school-aged children with SLD in a clinical or educational setting. These tools are currently used widely in practice. Four of these tools are presently available in the Occupational Therapy and Physical Therapy: A Resource and Planning Guide, 2nd edition (19) for use by clinicians, occupational and physiotherapists, special education teachers, and/or parents. This review describes the characteristics of five tools and compares their strengths and limitations. The selected tools include: i) BOT-2; ii) MABC-2; iii) PBS; iii) VABS-II; iv) PEDI-CAT.

\section{Methods}

Firstly, a comprehensive search of the literature has been conducted to identify existing paediatric tools. These tools are then evaluated to determine whether they meet the criteria for inclusion: i) standardised; ii) assess movement and/or function; iii) applicable to children with SLD ranging 4 to 17 years of age and iv) widely used in practice. Studies have been excluded if they are not currently available or if the review failed to find any literature on the tools. Finally, once the tools were included for review, complimentary publications were searched to enable a thorough evaluation of the tools. The selected studies describe the tools and their advantages or limitations in assessing children with SLD.

\section{Data Sources and Searches}

A literature review was conducted using the following databases: Medline, PubMed, EBSCOhost, OVID, ERIC and Google Scholar. The review looked at publications from 2000 to 2015. The first search used the following search term: Movement or gross motor or fine motor or balance and function or activity of daily living and assessment or evaluation or instrument and children or school-aged children and learning disabilities or learning disorders or SLD or dyslexia. The second search was performed to find supporting publications required for a further investigation of the selected tools. The search terms Identified assessment tool and learning disabilities or learning disorders or dyslexia have been used to search the available literature related to each tool. The flowchart of the article selections is shown in Figure 1. 
Review Article | Standardised movement and function assessment tools
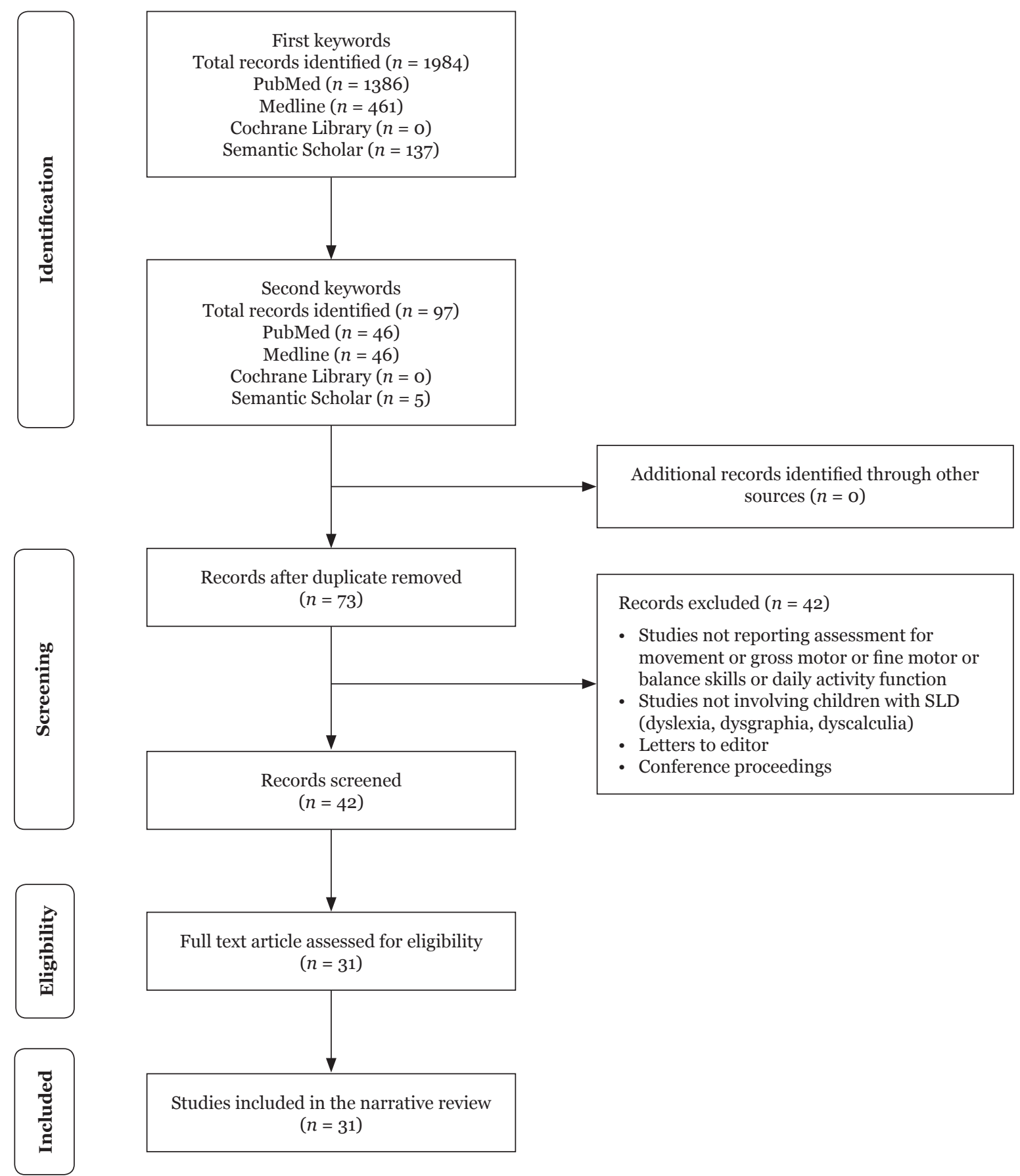

Figure 1. Flow chart of study selection

\section{Results}

\section{Characteristics of Movement and/or Function Assessment Tool}

Five tools that meet the study's inclusion criteria are: i) BOT-2; ii) MABC-2; iii) PBS; iv) VABS-II and v) PEDI-CAT.
The administrative aspects of the reviewed tools such as the domains, age of children, duration of test administration, specific training required, administrator criteria, origin and cost are presented in Table 1. 


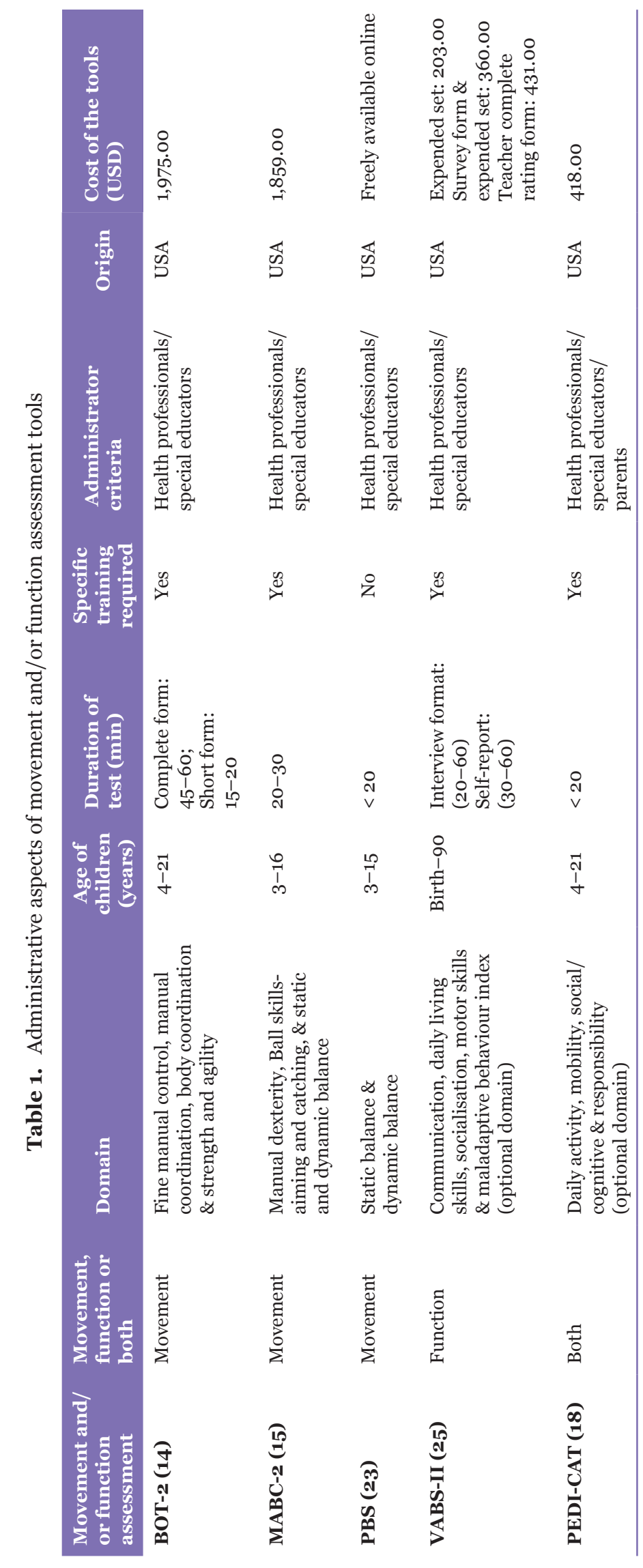


Review Article | Standardised movement and function assessment tools

BOT-2

\section{Description}

The BOT-2 (14), a revised version of the Bruininks-Oseretsky test of motor proficiency (BOTMP) (20), is used by education and health professionals to measure the performance of gross and fine motor skills in children aged 4-21 years. The test is proposed as both a screening tool and a diagnostic tool for children who may have motor impairments. It is also used for student selection in school placement and as an evaluative measure of the effectiveness of an intervention in movement and functional skills performance.

This tool consists of BOT-2 complete and short form. There are four motor area composites covered in the BOT-2 complete form including: i) fine manual control or FMC (subtests: fine motor precision and fine motor integration); ii) manual coordination or $\mathrm{MC}$ (subtests: manual dexterity and upper limb coordination); iii) body coordination (BC) (subtests: bilateral coordination and balance) and iv) strength and agility (SA) (subtests: running speed and agility and strength). However, the BOT-2 short form comprises 14 test items that are proportionately selected from the complete form.

The FMC refers to motor skill activities involving control and coordination of the distal musculature of the hands and fingers, such as cutting and copying shapes. The MC refers to motor skill activities involving control and coordination of the arms and hands, such as stringing blocks and dribbling a ball. The $\mathrm{BC}$ refers to control and coordination in posture and balance used by the large muscle groups such as jumping in place and standing one leg on a beam. Meanwhile, the SA covers aspects of fitness and coordination required in physical activity, such as running and sits-up.

Equipment and materials for administration the assessment tool, such as a manual, easel, record form and exam booklet, are provided with the purchase of a kit. The time required to administer the complete form varies from $45 \mathrm{~min}-60 \mathrm{~min}$, whereas the short form takes about 15 min-20 min. An additional 10 min of preparation is required before conducting the assessment.

\section{Administration of the Test}

The examiner is required to prepare the administration area before conducting the test. All required materials are placed accordingly and the child's hand and foot preferences are determined. To begin the test, the examiner needs to follow all the administrative rules and teaching tasks. An optional administration book containing an image of a child performing the task may be placed in front of the person taking the test. The examiner uses verbal instructions and is allowed to demonstrate or provide support if necessary.

\section{Scoring Procedures}

First, raw scores are obtained for each subtest, before each raw score is converted into a scale and a standard score. All information is obtained from the record book. Next, the sum of scores of four motor composites, i.e. $\mathrm{FMC}, \mathrm{MC}, \mathrm{BC}$ and $\mathrm{SA}$, form a total motor composite. Total scores are then converted into an equivalent motor age and into descriptive categories (classification) of motor performance. Another section in the record book is the score profile. A confidence interval (90\% and 95\%) can be plotted by referring to the scale scores and standard scores. Otherwise, the pair-wise comparisons can be examined for statistical significance and frequency of difference.

\section{MABC-2}

\section{Description}

The MABC-2 (15), a revised version of the movement assessment battery for children (MABC) (21), is used to identify motor impairment and to provide a description of motor difficulties in children. It is also the test that is most frequently used by examiners to test the gross motor performance in children. The test is intended for use by both education and health professionals (22).

There are two forms of the MABC-2 that comprise the performance test and the checklist. The performance test is designed to assess fine and gross motor skill movement difficulties in children aged 3 to 16 years in three different age bands: i) Band 1: 3-6 years; ii) Band 2: 7-10 years and iii) Band 3: 11-16 years. Conversely, the checklist is used by parents, caregivers or teachers to rate how a child manages everyday tasks encountered at home and in school. 
Both forms are useful for identifying and describing motor function in children.

The MABC-2 contains three sections, which are manual dexterity (three items), ball skillsaiming and catching (two items), and static and dynamic balance (three items). The test kit consists of an intervention manual, checklists, record form, test materials and additional required equipment such as chairs, a table and a clipboard. Twenty to forty minutes to administer the performance test, while the checklist takes about 10 min to administer.

\section{Administration of the Test}

To conduct the test, the examiner follows the task sequence according to the age bands in the examiner manual. However, the examiner may change the task order to maintain the interest and motivation of the children. The examiner is required to leave a note on the record form for future reference if the order is changed. Qualitative information on how the child approaches and performs the task is provided in the performance test. Trials for every task performance are given to the examinee before performing the task.

\section{Scoring Procedures}

The first step in scoring the test items is to record the raw performance score. A score of ' $F$ ' is given if the child fails to complete the task, 'I' if the task is inappropriate, or ' $R$ ' if the child does not cooperate. Next, the raw performance score of each item is then converted into a standard score, with low standard scores indicating poor performance. However, according to the checklist high scores represent poor performance.

\section{PBS}

\section{Description}

The PBS (23), a paediatric version of Berg's balance scale, is used to assess static and dynamic balance in school-age children aged $3^{-15}$ years with mild-to-moderate motor impairment. The test is intended for use by education and healthcare professionals for screening and evaluation.

This tool consists of 14 items pertaining to static and dynamic balance. The PBS requires equipment commonly found in schools and clinics, such as a height-adjustable bench, a chair with an armrest and back support, and a stopwatch. The test can be administered and scored in less than $20 \mathrm{~min}$.

\section{Administration of the Test}

To conduct the test, the examiner must demonstrate each task and explain the instructions that are given on the scoring sheet. The test allows for multiple trials of each item, and for the examinee to clarify the tasks both verbally and visually.

\section{Scoring Procedures}

A score ranging from $0-4$ is given for each item. The children are allowed for multiple trial on many of the items. The results should be rated according to the lowest standard that define the best performance of the child. For example, if during the first trial a child receives a maximum score of 4, then an additional trial need not be administered. The examiner may also choose to record the exact time in seconds for some scoring items. For items that pertain to balance, the subject is allowed to choose their preferred standing leg. Likewise, for items pertaining to reach, the subject is allowed to determined how far to stretch. A good balance performance is indicated by a higher score (24).

\section{VABS-II}

\section{Description}

The VABS-II (25) is a revision of the Vineland adaptive behaviour scale (Vineland ABS) (26). The tool is an individually administered instrument that measures a person's adaptive level of functioning, which is used for both diagnostic and evaluative purposes. It is available in four forms, which are the survey interview form, the expanded interview form, the parent/caregiver rating form and the teacher rating form (TRF). This assessment is used from infancy to 90 years of age, except for TRF which is applicable to the age ranges of $3-21$ years. The adaptive behaviour domain is conceptualised as encompassing the four broad dimensions of i) communication: receptive, expressive, written; ii) daily living skills: personal, domestic, community; iii) socialisation: interpersonal relationships, play and leisure time, coping skills; and iv) motor skills: fine motor and gross motor. In addition, the VABS-II also includes a maladaptive behaviour index: internalising and externalising, which the examiner may optionally assess. 
Review Article | Standardised movement and function assessment tools

The VABS-II takes approximately $20 \mathrm{~min}-$ 60 min to be administered, depending on the adaptive levels exhibited by the person assessed. An additional $15 \mathrm{~min}-30 \mathrm{~min}$ are needed to hand-score the instrument.

\section{Administration of the Test}

To conduct the test, the parents and/or caregivers are administered a semi-structured interview for the interview form (both survey and expanded). In contrast, both rating forms (parent/caregiver and teacher) need to be filled out independently using a provided rating scale.

\section{Scoring Procedures}

A score of $\mathrm{o}$ indicates that the person never performs the skills independently, while 1 indicates that they 'sometimes or partly perform skills independently' and 2 indicates that they 'often perform skills independently'. Individual items are rated. All sub domain item scores are totalled and raw scores are transformed into a scale score, domain score and adaptive behaviour composite.

\section{PEDI-CAT}

\section{Description}

The PEDI-CAT (18) is a revision of the pediatric evaluation of disability inventory (27) into a computerised-adaptive test (CAT) and is used to measure the functional skills of children and youth from infancy to 20 years of age. The PEDI-CAT is designed for use by health professionals, such as occupational therapists and physiotherapists, as well as professional educators in schools. The test is specifically designed for some clinical use, for example, a screening tool to detect functional delay, or as an evaluative measure and observation of individual change in a child. There are two types of PEDICAT, which include speedy (precision) CAT and content-balanced (comprehensive) CAT. The speedy CAT gives the fastest results as it consists of $5^{-15}$ items per domain, whereas the contentbalanced CAT consists of approximately 30 items per domain. There are a total of 271 items in four domains, including daily activities, mobility, social/cognitive and responsibilities. The PEDICAT requires equipment such as a computer or iPad installed with CAT software, a table, and a chair. It takes approximately $15 \mathrm{~min}-20 \mathrm{~min}$ to complete the items in the content-balanced CAT assessment.

\section{Administration of the Test}

Parents/caregivers can complete the PEDI-CAT independently through a structured interview or by professional judgment. To begin the test using the Windows version, the interviewer must first enter the identification number of the child and select the domain that is being assessed. Then, the interviewer selects the required demographic information such as gender, date of birth and types of mobility. Next, the interviewee must respond to the items that appear on the screen. About 20 min are required to complete all questions.

\section{Scoring Procedures}

The test uses a 4-point difficulty scale with a response of 1 indicating 'unable', 2 indicating 'hard', 3 indicating 'a little hard' and 4 indicating 'easy'. The original PEDI is scored using a twopoint response: 'unable or capable'. The PEDICAT provides two types of summary scores calculated for each domain. A normative score is provided as age percentile and $\mathrm{T}$ score. These results are based on a child's chronological age and the child's functioning is interpreted in relation to others of the same age. A scaled score provides the current functional skills of a child and progress over time. Additionally, an item map is presented if the subject is using a content-balanced CAT. The map will represent a sequential pattern of functional skills consistent with a child development.

\section{A Review of the Strengths of the Movement and/or Function Assessment Tools}

The strengths of the tools include their reliability and validity, cross-cultural applications, standardisation, and special features/characteristics. A summary of the strengths is presented in Table 2.

\section{Reliability and Validity}

Generally, evidence shows that a lack of information exists on the reliability and validity of the selected tools for children with SLD. However, the review of the five tools, i.e. BOT-2, MABC-2, PBS, VABS-II and PEDI-CAT demonstrates that the tools have some strong psychometric properties. 


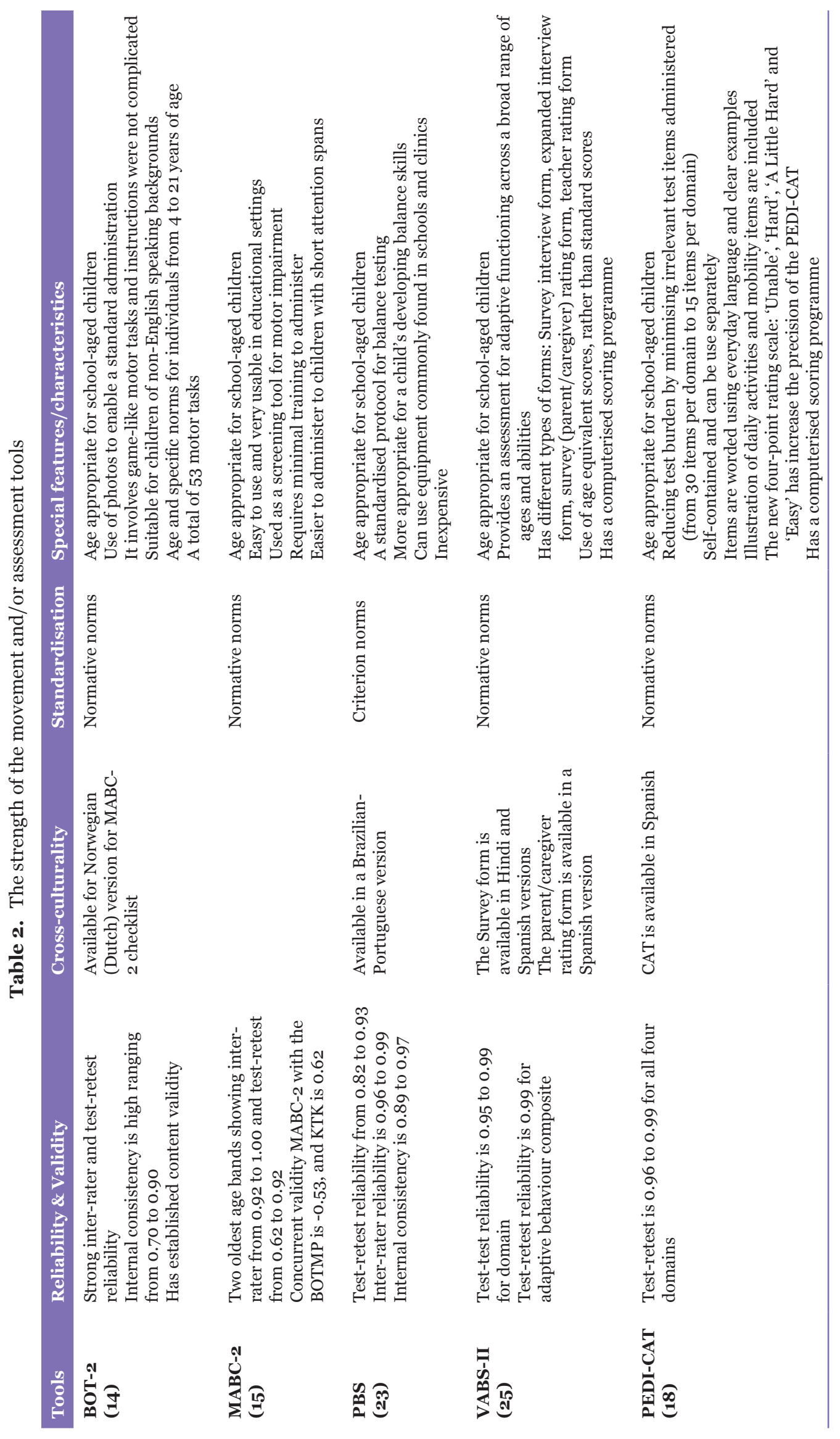


Review Article | Standardised movement and function assessment tools

Findings from a reliability study show that the BOT-2 is reported to have moderate to strong inter-rater and test-retest reliability for both complete and short forms (28). The BOT-2 shows split-half reliability for internal consistency and reliability coefficients for the subscale, composite, total motor composite and short form scores that range from high $0.70 \mathrm{~s}$ to mid-0.90 s (28).

For MABC-2, in the two oldest age bands, the inter-rater and test-retest reliability shows an intra-class correlation (ICC) range from $r=0.92-1.00$ and between $r=0.62$ and 0.92 , except for one item (29).

Similarly, the PBS has good reliability in assessing balance with excellent test-retest reliability, i.e. the ICC coefficient is $0.82-0.93$, inter-rater reliability, ICC is $0.96-0.99$ and internal consistency, Cronbach's $\alpha$ is $0.89-0.97$ (23).

The test-retest reliability of VABS-II has been found to be high: $r=0.95^{-0.99}$ for all domains and adaptive behaviour composite showing ICC $=0.99 ; r=0.74(n=160,6-18$ years, standardisation sample) is reported in Sparrow et al. (26).

For PEDI-CAT, previous studies have shown that test-retest reliability for all four domains is high (ICC $=0.96-0.99$ ) (30). However, the reliability of the reviewed assessment tools is well developed compared to its validity.

Content and concurrent validity is the common validity that has been established. For example, the BOT-2 has established content validity (31). For MABC-2, the concurrent validity with the BOTMP $r=-0.53$ and with Korperkoordinationtest fur Kinder (KTK) $r=0.62$ (32).

\section{Cross Cultural Applicability}

The Dutch translation of the MABC-2 checklist shows a construct validity with a Cronbach's alpha score of 0.94 (33). The total score of the Brazilian-Portuguese PBS version also shows excellent intra-rater reliability with an ICC, 0.85 and inter-rater reliability with an ICC, 0.91 (24). In addition, the survey form of VABS-II was translated into a Hindi version and has strong validity and reliability (34). A Spanish version of the survey interview form and parent/caregiver rating form is also available (35). PEDI has been translated into multiple languages, including Dutch, Norwegian, Swedish, Spanish (United-States), Portuguese (Brazil),
Slovene, Turkish, Icelandic, French (Canada), Hebrew, Japanese and Chinese (17). PEDI-CAT is available in Spanish.

\section{Standardisation}

Most of the assessments in the review have normative norms, which are represented by the United States population (BOT-2, VABS, PEDI-CAT) and European children (MABC-2). The PBS refers to criterion norms (34). The standardisation helps to determine movement and/or functional performance of a child individually and in relation to the general population at a similar age.

\section{Special Features or Characteristics}

There are a few special features or characteristics of the tools that can be observed in this review.

\section{Age specificity}

All tools were appropriate for school-aged children ranging from 4-17 years of age.

\section{The use of photos in the test}

The BOT-2 is a standardised tool that provides photos in each subtest to allow for a standard and effective administration of the test (28). This tool has proven to be transparent and relevant to childhood motor activities such as ball skills, movement, paper/pencil tasks, and card sorting (28).

\section{The user-friendly assessment tool}

The MABC-2 is designed to be user friendly, is easy to administer, and is very applicable to educational settings (36). It is recommended that the MABC-2 be used as a screening tool for motor impairment due to its simple test administration (37-38). Moreover, the MABC2 requires minimal training and is commonly used due to its sound psychometric properties (39). Furthermore, a short administration time (20 $\mathrm{min}$ ) is an advantage for children with a short attention span, and therefore, this test is widely used to measure motor performance (40). The game-like motor tasks for BOT-2 are able to capture the child's interest and the verbal instructions are easily understood (31). Therefore, the BOT-2 is suitable for children who do not speak English as a native language. The complete version of BOT-2 has a total of 53 items, compared to 46 items in BOTMP (39) Therefore, a wider range of motor tasks can be evaluated using this tool, including gross motor, fine motor and balance skills. 


\section{Less costly assessment tool}

The PBS is one of the standardised protocols for testing the balance of children with disabilities. The tool is able to distinguish between children who are developing normally and children with mild motor impairments (41). This test is inexpensive, can be downloaded at no cost, and the equipment needed to administer the test is easily available in schools, clinics, or hospital settings (42).

\section{Broad sensitivity for ages and abilities}

The VABS-II provides an assessment of adaptive functioning across a broad range of ages, i.e. from $0-90$ years old and is suitable for school-aged children (24). The VABS-II provides different forms for teachers and parents to report any developmental problems that children may have (35). This tool also has a broad sensitivity across ages and ability levels. Age-equivalent scores are used, which provides an advantage when comparing performance domains.

\section{Computerised-scoring programme}

The VABS-II consists of a computerisedscoring programme that makes the computation and interpretation of scores much easier and may avoid problems that can arise in manual scoring. The scoring instructions are nicely formatted and easy to follow and the manual contains several examples of completed protocols with annotations of how to discontinue standards, how to calculate raw scores on each scale and how standardised scores are obtained based on raw scale scores. The PEDI-CAT is selfcontained, and can be used to assess separate domains such as daily activities, mobility, social/cognitive and responsibilities (43). The PEDI-CAT is designed with clear and simple instructions, and illustrations are provided for each item to improve clarity (43). Finally, the new 4-point rating scale: 'Unable', 'Hard', 'A little hard' and 'Easy', has increased the precision of the PEDI-CAT rather than 'capable/ not capable' in the old version of PEDI (43).

\section{A Review of the Limitations of the Movement and/or Function Assessment Tools}

The limitations highlight the examiner, the administration of the test, and the scoring procedures. The summary of the limitations for the five reviewed tools are presented in Table 3.

\section{Examiner}

The biggest limitation pertaining to the examiners is that they are required to have high qualifications or training to use some tools, especially to score and interpret the results. Some also report that a tool such as the BOT-2 is hard to obtain $(31,44)$.

\section{Administration of the Test}

In using BOT-2 to test a shuttle run, an open/large room is needed in order to complete the 50-foot shuttle run in under $13 \mathrm{~s}$ and this can be a challenge $(31,44)$. Moreover, the time required to complete one comprehensive test in BOT-2 is rather long (45 $\mathrm{min}-60 \mathrm{~min}$ ). This may pose a challenge to children below three years of age who are participating in the assessment (39, 44).

MABC-2 requires that only the equipment supplied in the test kit be used. Any change may invalidate the results obtained (40). In addition, the tool does not cover the full range of motor abilities in children due to the fact that there are only eight tasks in each domain being assessed (12). Furthermore, the time duration of administration (20 min-30 min for eight items) is an unacceptable proportion and is too long (45). This test is also limited to certain age bands and age skills (45). Finally, repeated trials (5 practice and 10 test trials) can be a challenge for some children who may become too tired to perform the tasks. A longitudinal analysis of MABC-2 is difficult to conduct due to different tasks and age bands (46). Furthermore, there are no separate norms for boys and girls in MABC-2 (46).

The PBS is influenced by height. Examples of this barrier are found in the items sit to stand, transfers, turning to look behind, retrieving an object from the floor and reaching forward (42). During the transfer technique, most children with a shorter statue will wiggle back into their chair. Therefore, it is not possible for these children to achieve a full score for this item. The PBS also tests static balance but not locomotive balance or overhead reaching (42). Therefore, dynamic balance cannot be tested using this tool.

The VABS report does not provide a selfreport form and is lengthier than some other measures of adaptive functioning (24).

The reported assessment limitations using PEDI-CAT include items in the responsibility domain that require children to use a combination of functions to carry out life tasks 
Review Article | Standardised movement and function assessment tools

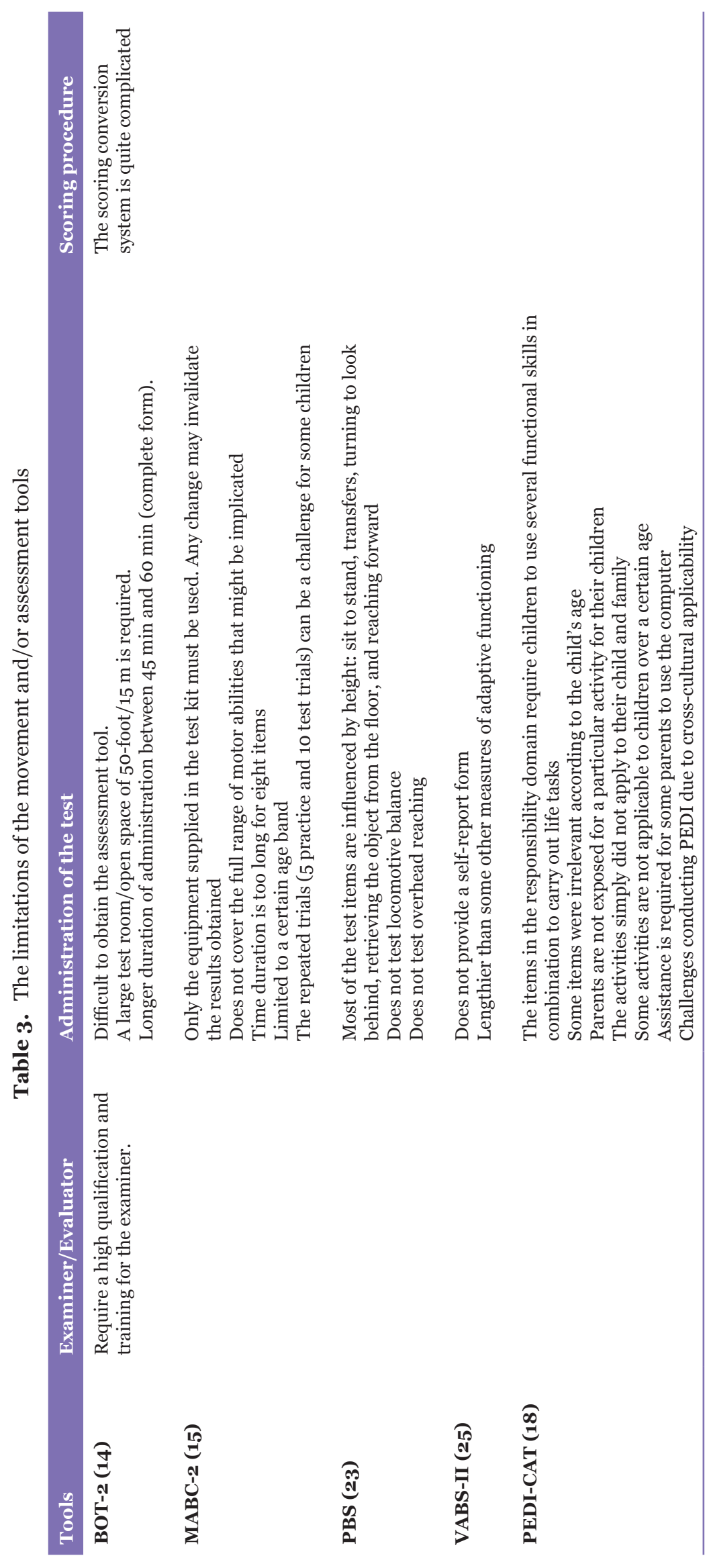


irrelevant of their age. They also include tasks that do not allow a particular activity to be completed, and certain tasks that are simply too difficult for younger children to complete. Furthermore, certain activities simply do not apply to a particular child and family, and are not applicable to older children (30). For this reason, parents may find this domain and it's given questions difficult to respond to. Moreover, some parents may require assistance in using the computer (30). There is also a lack of information on the cross-cultural differences that exist around using the tools in children with SLD. Many studies have reported challenges conducting PEDI due to issues of cross-cultural applicability i.e. problems with language translation, cultural differences in some activities, and different beliefs pertaining to encouraging a child to perform certain activities (17). Additionally, one of the largest difficulties regarding translation is finding suitable words in different languages. For example, Berg et al. (47) report difficulty in translating 'prompting', 'fasteners' and 'item' into Norwegian words. In addition, the use of a bathtub is not common in some countries, as reported by Norwegian and Dutch teams (17). Lastly, different parenting experiences in normative data between America and other countries produce different results (47).

\section{Scoring Procedures}

Previous studies report that the scoring conversion system in BOT-2 is quite complicated and may provide a challenge to the examiner during the scoring process $(28,39)$. This is particularly true with regard to confusion that arises when using the BOT-2 record form and test manual (28).

\section{Discussion}

To date, there are many assessment tools available to assess the movement and/or function of children with learning disabilities $(14-15,17-18)$. Overall, each assessment tool in the articles reviewed was used to measure the movement or functional skills performance of school-aged children with SLD. Only the PBS is freely available online, while other assessments must be bought at a fairly expensive price with an authorised dealer and need training to administer. All recent assessment tools also were developed in the English language. Therefore, thorough translation work and validation studies must be conducted before such tools are used in different cultural contexts (48). Several first spoken languages exist in Malaysia including Bahasa Melayu, Chinese, Tamil, Kadazan-Dusun and Jaku-Iban (49). Therefore, a functions assessment tool, which usually requires parents to use a rating scale, may pose a challenge to some parents who may not be able to understand or interpret the item tasks.

These five assessment tools were standardised and have strong reliability and validity. Some of the assessment tools were translated to other ethnic-language version such as Norwegian (Dutch), Brazilian (Portuguese), Hindi and Spanish. Certain unique features were designed for each tool, such as graphics, gamelike motor tasks and user-friendly aspects, to ensure that the tools were useable for children with learning disabilities.

Unfortunately, some limitations exist regarding the administration of certain tools, which may disadvantage some people if they used to assess the movement and functional skills performance of children with SLD. For example, the BOT-2 is very difficult to obtain and a large space is needed to conduct the assessment. Item tasks in the MABC-2 and VABS-II also require a lot of time and repeated trials, which were not appropriate especially for young children. This review recommends that assessment tools with a shorter administration time are used with young children, such as pre-school aged children. A long test duration may pose challenges to younger children in participating throughout the duration of the assessment (45), and the test may have to be administered over multiple sessions due to a child's fatigue. Some item tasks in the PEDI-CAT were irrelevant according to age, not culturally relevant, or the child do not have the opportunity to participate to the activity yet. Therefore, this may pose challenges to parents who are rating the scores of these tests.

Many different factors influence the selection of assessments of children with SLD. According to Cools et al. (45), certain criteria for the selection of assessments of children should be considered, such as the purpose of assessment. For example, the evaluative measure of general motor proficiency, fine or gross motor proficiency assessment, and the prevalence of assessment must be specified. Secondly, the test must be appropriate and specific to the target 
Review Article | Standardised movement and function assessment tools

population. Moreover, the test must be easy to understand and administer. Lastly, differences exist between the assessments regarding cultural norms and cultural similarities. Therefore, this review recommends using a suitable assessment tool which is standardised, relevant and specific to the target population, and one that can be understood by the raters and acceptable to the specific population norms.

\section{Conclusion}

In conclusion, the primary goal of the review is to present the characteristics, strengths and limitations of five recent standardised assessment tools in practice, which measure the movements and/or functional skills of children with SLD. A summary of the characteristics, strengths and limitations of these assessment tools can be found in Table 3 .

\section{Acknowledgements}

None.

\section{Ethics of Study}

None.

\section{Conflicts of Interest}

None.

\section{Funds}

This research was funded by the Ministry of Science, Technology and Innovation (MOSTI) Malaysia (06-01-02-SF1233).

\section{Authors' Contributions}

Conception and design: NSB, DH

Analysis and interpretation of the data: NSB

Drafting of the article: NSB

Critical revision of the article for important

intellectual content: NSB, DH, MK

Final approval of the article: NSB, DH, MK

Obtaining of funding: $\mathrm{DH}$

\section{Correspondence}

Dr Dzalani Harun

PhD (Universiti Kebangsaan Malaysia)

Occupational Therapy Programme,

Center for Rehabilitation and Special Needs

Studies, Faculty of Health Sciences,

Universiti Kebangsaan Malaysia,

Jalan Raja Muda Abdul Aziz,

50300 Kuala Lumpur, Malaysia.

Tel: +60392897914

Fax: +603 26914304

E-mail: dzalani@ukm.edu.my

\section{References}

1. American Psychiatric Association. Diagnostic and statistical manual of mental disorders (DSM5). 5th ed. Washington, DC, USA: American Psychiatric Association; 2013.

2. Hock TT, Chan WS, Abdullah MR. Clinical diagnosis and non-verbal ability of primary-one school children with learning disabilities. Int $J$ Public Health Res. 2011;6:33-40. https://doi .org/10.25291/VR/33-VR-40

3. Fouzia KA. Exploring the invisible: issues in identification and assessment of students with learning disabilities in India. Transcience. 2015;6:91-107.

4. Mayes SD, Calhoun SL. Test of definition of learning disability based on the difference between IQ and achievement. Psychol Rep. 2005;97:109-116. https://doi.org/10.2466/pro .97.1.109-116

5. Madhuri K, Sarika K, Shubhangi U, Sunil K, Sanjeev A. Approach to learning disability. Indian J Pediatr. 2001;68:539-546. https://doi. org/10.1007/BFo2723250

6. Deval S, Writer H, Mate K. Assessment of gross motor proficiency in children with learning disability. Indian $J$ Physiother Occup Ther. 2013;7:229-234. Retrieved from http://www.isholar.in/index.php/ijpot/article/view/43360

7. Capellini AS, Coppede C, Valle TR. Fine motor function of school-aged children with dyslexia, learning disability and learning difficulties (original title: Funcao motora fina de escolares com dislexia, disturbio e dificuldades de aprendizagem). Pro-Fono. 2010;22:201$208 . \quad$ https://doi.org/10.1590/So10456872010000300008 
8. Rochelle KSH, Talcott JB. Impaired balance in developmental dyslexia? A meta-analysis of the contending evidence. $J$ Child Psychol Psychiatr. 2006;47:1159-1166.

9. Stoodle CJ, Stein JF. The cerebellum and dyslexia. Cortex. 2011;47:101-116. https://doi. org/10.1016/j.cortex.2009.10.005

10. Okuda PMM, Ramos FG, Santos LCA, Padula NAMR, Kirby A, Capellini SA. Motor profiles of students with dyslexia. Psychoogy Res. 2014;4:31-39.

11. Nonis KP, Jernice TSY. The gross motor skills of children with mild learning disabilities. Int $J$ Spec Educ. 2014;29:92-97.

12. Staples KL, MacDonald M, Zimmer C. Assessment of motor behaviour among children and adolescents with ASD. 2012;42:179214. https://doi.org/10.1016/B978-0-12-394284-5 $.00007-3$

13. Lubans DR, Morgan PJ, Cliff DP, Barnett LM, Okely AD. Fundamental movement skills in children and adolescents: review of associated health benefits. Sports Med. 2010;40:1019-1035. https://doi.org/10.2165/11536850-000000000 $-00000$

14. Bruininks RH, Bruininks BD. BruininksOseretsky test of motor proficiency. 2nd ed. Minneapolis, USA: Pearson; 2005.

15. Henderson SE, Sugden DA, Barnett A. Movement assessment battery for children. 2nd ed. London: Harcourt Assessment; 2007.

16. Hinojosa J. Statement-fundamental concepts of occupational therapy: occupation, purposeful activity and function. Amer J Occupational Ther. 1997;51:864-866. https://doi.org/10.5014/ajot .51 .10 .864

17. Sparrow SS, Cicchetti DV, Balla DA. Vineland-II adaptive behavior scales: survey forms manual. Circle Pines, MN: AGS Publishing; 2005.

18. Haley S, Coster W. PEDI-CAT: Development, standardization and administration manual. Boston, MA: CRECare, LLC; 2010.
19. Bober P, Corbett S. Occupational therapy and physical therapy: a resource and planning guide. 2nd ed. Madison, WI: Wisconsin Department of Public Instruction; 2011.

20. Bruininks RH. Bruininks-Oseretsky test of motor proficiency: examiner's manual. Circle Pines, MN: American Guidance Service; 1978.

21. Henderson SE, Sugden DA. Movement assessment battery for children. London: Psychological Corporation; 1992.

22. Henderson SE, Barnett AL. The classification of specific motor coordination disorders in children: Some problems to be resolved. Hum Mov Sci. 1998;17:449-469. https://doi.org/10.1016/So167 $-9457(98) 00009-8$

23. Franjoine MR, Gunther JS, Taylor MJ. Pediatric balance scale: a modified version of the berg balance scale for the school-age child with mild to moderate motor impairment. Pediatr Phys Ther. 2003;15:114-128. https://doi.org/10.1097/01. PEP.0000068117.48023.18

24. Ries LG, Michaelsen SM, Soares PS, Monteiro VC, Allegretti KM. Cross-cultural adaptation and reliability analysis of the Brazilian version of pediatric balance scale (PBS). Braz J Phys Ther. 2012;16(3):205-215. https://doi.org/10.1590/ S1413-35552012005000026

25. Sparrow S, Cicchetti D, Balla D. Vineland adaptive behavior scales. Circle Pines, $\mathrm{MN}$ : American Guidance Service; 2005.

26. Sparrow S, Balla D, Cicchetti D. Vineland adaptive behavior scales: survey forms manual. Circle Pines, MN: American Guidance Service; 1984 .

27. Haley S, Coster W, Ludlow L, Haltiwanger J, Andrellos P. Pediatric evaluation of disability inventory (PEDI): development, standardization and administration manual. Boston, MA: Trustees of Boston University; 1992.

28. Deitz JC, Kartin D, Kopp K. Review of the Bruininks-Oseretsky test of motor proficiency (BOT-2). Phys Occup Ther Pediatr. 2007;27(4):87-102. https://doi.org/10.1080/ Joo6v27no4_o6 
29. Chow S, Henderson S. Interrater and test-retest reliability of the movement assessment battery for Chinese pre-school children. Am J Occup Ther. 2003;57:574-577. https://doi.org/10.5014/ ajot.57.5.574

30. Dumas HM, Fragala-Pinkham MA, Haley SM, Ni P, Coster W, Kramer JM, et al. Computerize adaptive test performance in children with and without disabilities: prospective field study of the PEDI-CAT. Disabil Rehabil. 2012;34:393-401.

31. Lucas BR, Latimer J, Doney R, Ferreira ML, Adams R, Hawkes G, et al. The Bruninksoseretsky test of motor proficiency-short form is reliable in children living in remote Australian aboriginal communities. BMC Pediatr. 2013;13:1-14. https://doi.org/10.1186/1471-2148$13-135$

32. Croce RV, Horvat M, McCarthy E. Reliability and concurrent validity of the movement assessment battery for children. Percept Mot skills. 2001;93:275-280. https://doi.org/10.2466/ PMS.93.5.275-280

33. Schoemaker MM, Niemejer AS, Flapper BC, Smits-Engelsman BC. Validity and reliability of the movement assessment battery for children-2 checklist for children with and without motor impairments. Dev Med Child Neurol. 2012;54:368-375.

34. Kumar RS, Shankar K, Kush V, Kumar C, Bhave A, Agarwal V. Adaptation: Vineland adaptive behavior scale for 3-9 year-old Indian children. Int $J$ Disabil Hum Devt. 2015;15:49-55. https://doi.org/10.1515/ijdhd-2014-0026

35. Community-University Partnership for the Study of Children, Youth, and Families. Review of the Vineland adaptive behavior scales. 2nd ed (Vineland-II). Edmonton, Alberta, Canada; 2007.

36. Brown $\mathrm{T}$, Lalor A. The movement assessment battery for children-(MABC-2): a review and critique. Phys Occup Ther Pediatr. 2009;29:86-103. https://doi.org/10.1080/ 01942630802574908

37. Liu T, Hamilton M, Smith S. Motor proficiency of the head start and typically developing children on MABC-2. J Child Adolesc Behav. 2015;3:198. https://doi.org/10.4172/2375-4494.1000198
38. Petermann F. Movement assessment of battery for children-2 (MABC-2). Frankfurt: Pearson PLC; 2008.

39. Piek JP, Hands B, Licari MK. Assessment of motor functioning in the preschool period. Neuropsychological Review. 2012;22:402-413. https://doi.org/10.1007/s11065-012-9211-4

40. Spironello C, Hay J, Missiuna C, Faaught BE, Cairney J. Concurrent and construct validation of the short form of the Bruininks-Oseretsky test of motor proficiency and the movement-ABC when administered under field conditions: implications for screening. Child: Care, Health and Dev. 2010;36:499-507. https://doi.org/10.1111/j.13652214.2009.01066.x

41. Darr N, Franjoine MR, Young B, Abbot H, Basso G, Brennan A, et al. Pediatric balance scale performance in children who are developing typically and in children with mild developmental delays. Pediatr Phys Ther. 2009;21:89-90

42. Franjoine MR, Darr N, Held SL, Kott K, Young BL. The performance of children developing typically on the pediatric balance scale. Pediatr Phys Ther. 2010;22:350-359.

43. Kao YC, Kramer JM, Liljenquist K, Tian F, Coster WJ. Comparing the functional performance of children and youths with autism, developmental disabilities, and no disability using the revised pediatric evaluation of disability inventory item banks. Am J Occup Ther. 2012;66:607-616. https://doi.org/10.5014/ajot.2012.004218

44. Peerlings W. Bruininks-Oseretsky test of motor proficiency. 2nd ed. In: Johan Simons, editor. Actuele Themata uit de psychomotorische therapie. Acco, Leuven/Leusden; 2007.

45. Cools W, Martelaer KD, Samey C, Andries C. Movement skill assessment of typically developing pre-school children: a review of seven assessment movement skill assessment tool. $J$ Sports Sci Med. 2009;8:154-168. https://doi.org/10.1249/o1. mss.0000354138.08376.d2

46. Barnett AL. Motor assessment in developmental coordination disorder: from identification to intervention. Int $J$ Disabil, Dev Educ. 2008;55:113-129. 
47. Berg M, Jahnsen R, Froslie K, Hussain A. Reliability of the pediatric evaluation of disability inventory (PEDI). Phys Occup Ther in Pediatr. 2004;24:61-77.

48. Coster WJ, Mancini MC. Recommendations for translation and cross-cultural adaptation of instruments for occupational therapy research and practice. Revista de Terapia Ocupacional da Universidade de São Paulo. 2015;26(1):50.
49. Balakrishnan B, Chong HB, Idris MZ, Othman AN, Wong MF, Azman MN. Culturally responsive multimedia tool framework for dyslexic children in Malaysia: a preliminary study. Geographia online. Malaysian Journal of Society and Space. 2016;3:102-110. 